\title{
CONJUGACY OF FREE FINITE GROUP ACTIONS ON INFRANILMANIFOLDS
}

\author{
by MICHAE SADOWSKI
}

(Received 14 December, 1988; revised 23 March, 1989)

In this note we give the proof of the following result (previously known for homotopically trivial and free actions on infranilmanifolds [3, Theorem 5.6]).

Theorem 1. Let $G$ be a finite group acting freely and smoothly on a closed infranilmanifold $M$. Assume that $\operatorname{dim} M \neq 3,4$. Then the action of $G$ is topologically conjugate to an affine action.

The following notions are used here. A diffeomorphism $f$ of a Lie group $N$ onto a Lie group $N^{\prime}$ is said to be affine if $f=L_{g} \circ \Phi$, where $\Phi: N \rightarrow N^{\prime}$ is an isomorphism, $g \in G$, and $L_{g}: N^{\prime} \rightarrow N^{\prime}$ is given by $L_{g}(x)=g x$. An infranilmanifold is an orbit space $M=N / \Gamma$, where $N$ is a nilpotent simply connected Lie group, $\Gamma$ is a discrete group acting affinely, freely, and properly discontinuously on $N$ and such that $N \cap \Gamma$ has finite index in $\Gamma$. Note that $\Gamma$ is the deck group of $M$. This group is virtually nilpotent (that is $\Gamma$ is a finite extension of a nilpotent group). A diffeomorphism of one infranilmanifold onto another one is affine if it is covered by an affine diffeomorphism of nilpotent Lie groups.

Proof of Theorem 1. Since the group $G$ acts freely, the orbit space $M / G$ is a closed manifold. The group $\Pi_{1}(M / G)$ is virtually nilpotent. According to [1, Theorem 6.3], [2, Section 3.2, Corollary 1] there is an infranilmanifold $V_{0}$ and a homeomorphism $f_{0}: M / G \rightarrow V_{0}$.

If $p: M \rightarrow M / G$ is the canonical projection, then the following diagram commutes.

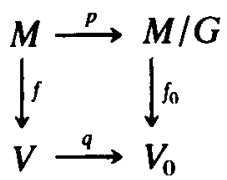

Here $q: V \rightarrow V_{0}$ is the covering induced by $f_{0}^{-1}$ and $f$ covers $f_{0}$. The map $q$ induces the structure of an infranilmanifold on $V$. For any $g \in G$ the homeomorphism $f \circ \sigma(g) \circ f^{-1}$ (where $\sigma(g)$ is the action of $g$ on $M$ ) induces the identity map on $V_{0}$ so that $f \circ \sigma(g) \circ f^{-1}$ is an affine transformation of $V$.

By [2, Section 4.2], there is an affine diffeomorphism $h: V \rightarrow M$. The formula $\rho(g)=h \circ f \circ \sigma(g) \circ(h \circ f)^{-1}$ defines an affine action of $G$ on $M$ that is topologically conjugate to the original action of $G$. The proof of Theorem 1 is complete.

REMARK 1 . The conjugating homeomorphism $h$ of can be chosen in such a way that it is homotopic to the identity, because every homeomorphism of a closed infranilmanifold is homotopic to an affine diffeomorphism ([2, Section 4]). 


\section{REFERENCES}

1. F. T. Farrell and W. C. Hsiang, Topological characterisation of flat and almost flat Riemannian manifolds $M^{n}(n \neq 3,4)$, Amer. J. Math. 105 (1983), 641-673.

2. Y. Kamishima, K. B. Lee and F. Raymond, The Seifert construction and its applications to infranilmanifolds, Quart. J. Math. Oxford 34 (1983), 433-452.

3. K. B. Lee and F. Raymond, Geometric realisation of group extension by the Seifert construction, Contemporary Math., 333 (1984), 353-411.

Department of Mathematics

THE UNIVERSITY OF GDAŃSK

80-952 Gdańsk, Wita Stwosza 57

Poland 